\section{Beta-Glucocerebrosidase Gene Mutations P.Asn409Ser and P.Leu483Pro in Polish Patients with Parkinson's Disease}

\section{Abstract}

Background: The aim of his study was to evaluate the frequency of two most frequent GBA gene mutations in heterozygote state in patients with early and later onset Parkinson's disease in Polish populations.

Methods and findings: Patients with Parkinson's disease; 115 non-demented with early onset disease ( $<45$ year-old) and 155 patients with onset over 45 year-old were recruited to the study. The p.Asn409Ser and p.Leu483Pro screening was performed with the PCR-RFLP and direct sequencing methods. In the group of 270 PD patients, 11 heterozygotes for mutations in the GBA gene were identified (carrier frequency $4.07 \%$ ). Among patients with early onset disease carrier frequency was $4.34 \%$ compared to $3,87 \%$ in the group of later onset.

Conclusion: The study yielded that carrier frequency of GBA mutation in polish population was comparable to other European populations p.Asn409Ser mutation dominates in patients with early onset PD disease.

Keywords: GBA Gene; Parkinson's Disease; Mutation; Dementia

Received: November 22, 2015; Accepted: December 19, 2015; Published: December 23, 2015

\section{Introduction}

Parkinson's disease (PD) is one of the most common neurodegenerative diseases with an incidence of 100-200 cases per 100,000 population. It belongs to the group of synucleinopathies and is characterized by slowness of movement, tremors, muscle rigidity, bradykinesia, and postural instability. Mutations in the SNCA, LRRK2, UCHL-1, PARK2, PINK1, and DJ-1 genes [MIM_163890, MIM_609007, MIM_191342, MIM_602544, MIM_608309, MIM_606324] are thought to be the pathogenic causes of familial PD. However, the pathomechanism of these sporadic forms of PD are not fully understood, although several explanatory hypotheses have been proposed.

It has been suggested that mutations in the GBA gene (MIM_606463) coding for lysosomal beta-glucocerebrosidase (GBA; EC 3.2.1.45) are a probable risk factor for PD and Levy body dementia (LBD) [1-4], but only one study has also linked it to other synucleinopathies [5]. The reported incidence of GBA gene mutations in PD patients has varied according to geographical area, population studied, methods of DNA testing (sequencing versus evaluating only the most common mutations), and control groups [6-9].
Zygmunt Jamrozik, Agnieszka Ługowska, Dariusz Kosiorowski, Andrzej Friedman, Jarosław Sławek, Piotr Janik, Anna Potulska-Chromik, Magdalena KuźmaKozakiewicz, Agnieszka Wiśniewska, Michał Szubiga, Monika Rudzińska, Andrzej Szczudlik and Dorota HZ

\section{Department of Neurology, Medical} university of Warsawa, Warsaw, Poland

Corresponding author:

Zygmunt Jamrozik MD, PhD

Đ zygmunt.jamrozik@wum.edu.pl

Department of Neurology Warsaw Medical University, Banacha 1 a Str, 02-093 Warsaw, Poland.

Tel: + 48-22-5992773

Fax: $+48-22-5991857$

Citation: Jamrozik Z, Ługowska

A, Kosiorowski D, et al. Beta-

Glucocerebrosidase Gene Mutations P.Asn409Ser and P.Leu483Pro in Polish Patients with Parkinson's Disease. J Neurol Neurosci. 2016, 6:4.

Beta-glucocerebrosidase is a lysosomal hydrolase located in the lysosomal membrane and involved in the degradation of a sphingolipid glucocerebroside (glucosylceramide, GlcCer). It is known that mutations in both alleles of the GBA gene lead to the Gaucher's disease (GD), an autosomal recessive metabolic disorder characterized by accumulation of undegraded GlcCer and secondary macrophage activation. The most frequent mutations 
causing GD are p.Asn409Ser and p.Leu483Pro (historical names N370S and L444P) [10].

Recent reports, however, suggest that mutations in the GBA gene present in a heterozygous state (i.e. in one allele of the gene) are associated with familial parkinsonism and an earlier age at the onset of PD [11-14]. Our study was conducted to evaluate the presence of the two most common GBA gene mutations in Polish patients with early onset PD.

\section{Material and Methods}

Two groups of PD patients were included in the study; a group of 115 non-demented PD patients with early onset of the disease ( $<45$ years of age) and a group of 155 patients with PD onset above 45 years of age. Patients were recruited based on the UK Parkinson's Disease Society Brain Bank Clinical Diagnostic Criteria. The presence of dementia was investigated in all PD patients according to the MMSE protocol.

To identify mutations in the GBA gene, genomic DNA was extracted from white blood cells using standard techniques. Molecular analysis was performed using restriction fragment length polymorphism analysis of polymerase chain reactionamplified fragments (PCR-RFLP) and direct sequencing methods in conditions excluding amplification of the $G B A$ pseudogene ( $p G B A)$. Screening for p.Asn409Ser and p.Leu483Pro mutations was performed in all patients as described in the literature (PCRPFLP with Ncil and Xhol, respectively) $[15,16]$. Additionally, in the group of later onset PD patients, sequencing of exons 2, 8, and 9 was performed (details on reaction conditions and starter sequences available on request), and the obtained sequences were compared to the rates reported for other general European populations and PD patients $[7,8]$.

\section{Results}

In the group of 115 PD patients with early onset of the disease, we identified 5 heterozygotes for the examined mutations in the GBA gene, including 4 individuals with and p.Leu483Pro and one person with $p . A s n 4095 e r$ (carrier frequency in this group was 4.34\%). In the later onset PD group, 6 of the 155 patients were heterozygotes for the $p$.Asn409Ser mutation (carrier frequency $3.87 \%$ ). Overall, 11 heterozygotes for mutations in the GBA gene were identified from the 270 PD patients in this study (carrier frequency $4.07 \%)$.
In the later onset PD group, the mean number of MMSE p.Asn409Ser mutation carriers was 26.2 (SD 3.7), similar to that obtained from patients without $G B A$ gene mutation $(X=27.4, S D$ 3.0, $\mathrm{P}=0.28$ ).

\section{Discussion and Conclusions}

The findings of this study demonstrate that the type of mutation in the GBA gene is strongly related to the age of PD onset and that most probably these mutations promote alpha-synuclein accumulation. The approximate carrier incidence of $4 \%$ found in our group of PD patients, independentage at onset of the disease, is about 10-fold higher as compared to the results obtained for a general European population, and similar to that reported earlier in PD patients (Table 1) [8].

In our group of PD patients, the L444P mutation was more frequently observed in individuals with an early onset of the disease, while the p.Asn409Ser mutation was more common in later onset PD patients, which is in line with what has previously been reported [17-19]. In patients with GD, the presence of the p.Asn409Ser mutation protects from neurological involvement, whereas homozygosity of the p.Leu483Pro mutation leads to the neuronopathic types 2 and 3 of GD. It seems that the presence of p.N370S in PD patients results in a later onset of the disease as compared to $p$.Leu483Pro carriers.

Alfa-synuclein is a protein encoded by the SNCA gene and expressed mainly in presynaptic terminals. Mutations in the SNCA gene lead to autosomal dominant familial PD. Alfa-synuclein binds to lipids present in plasma membrane, synaptic vesicles, and elsewhere. Under pathological conditions, alfa-synuclein aggregates to form various oligomeric structures and insoluble amyloid fibrils which characterize the synucleinopathies [20]. A number of different explanations for alfa-synuclein aggregation have been put forward including ones related to the role of proper lysosomal and autophagy functions, to reticulum-associated protein degradation (ERAD) system, and to influence of altered membrane lipid composition [21]. A prion-like hypothesis has been also been described, in which alfa-synuclein aggregates are transmitted through exocytosis and subsequent endocytosis between neighbouring cells (donors and recipients) and the misfolded protein then acts as a template to promote misfolding of alfa-synuclein in recipient cells [21,22]. Recently, Yap et al. have proposed that membrane-bound alfa-synuclein interacts with GBA, inhibiting its enzymatic function. Thus, mutations reducing GBA level/activity or interfering with alfa-synuclein may lead

Table 1 Comparison of the frequency p.L444P and p.N370S mutations in PD patients with early onset and later onset of disease.

\begin{tabular}{|c|c|c|c|c|}
\hline Mutation & Age at onset $<45$ years-old. & Age at onset $>45$ years-old & $\chi^{2}$ test with Yates' correction for continuity & Fisher's exact test two-sided \\
\hline L444P & 4 & 0 & $p=0.0673$ & $p=0.0319 *$ \\
\hline N370S & 1 & 6 & $p=0.2512$ & $p=0.2445$ \\
\hline Total L444P & & & $\chi^{2}$ test without Yates' correction & \\
\hline $\begin{array}{l}\text { total } \\
\text { N370S }\end{array}$ & 4 & 7 & $\begin{array}{c}\mathrm{p}=0.0343^{*} \\
\mathrm{p}=0.02143^{*} *\end{array}$ & $p=0.0152^{*}$ \\
\hline
\end{tabular}

Two-way table of frequencies *

Articulated table ** 
to reduced lysosomal degradation and result in alfa-synuclein aggregation as well as GlcCer accumulation, which together mediate an interaction with the protein-enzyme complex in vesicles leading to further loss of activity [23].

Although the effect of GBA gene mutations on PD development is undisputed, other pathological factors must also be considered in the pathomechanism of alfa-synuclein aggregation, since not all GD patients and GD carriers suffer from PD or parkinsonism. The low proportion of heterozygous carriers of the tested GBA mutations who develop PD suggests that heterozygosity for these mutations is neither the only nor the predominating factor in the pathophysiology of parkinsonism (Table 2).

The nonsignificant MMSE difference between the two patient subgroups in our study resulted from a relatively small patient sample and a small difference in the mean age between these groups. The study yielded that carrier frequency of GBA mutation in polish population was comparable to other European populations and p.Asn409Ser mutation dominates in patients with early onset PD disease.

\section{Acknowledgments}

The authors thank dr Jo Lewkowicz who edited our paper from the language point of view. The study was approved by Ethical Board Medical University of Warsaw; no KB/119/2008

\section{Competing Interests}

Jamrozik $Z$ and Ługowska A contributed equally in preparing manuscript. No other competing interests exist.

\section{Funding}

This work was supported in part by the Polish Ministry of Science and Higher Education (grant No. NN402 4404 33).

Table 2 Frequency of carriers for GBA mutations in different groups of patients with PD (partly from Velayati et al.).

\begin{tabular}{|c|c|c|c|}
\hline \multirow{2}{*}{ Population studied } & \multicolumn{2}{|c|}{ Carrier frequency } & \multirow{2}{*}{ Author and Year } \\
\hline & PD patients & Controls & \\
\hline Mixed & 21 & - & Lwin et al., 2004 \\
\hline Ashkenazi Jews & 31.3 & 6.2 & Aharon-Peretz et al., 2004 \\
\hline Ashkenazi Jews & 10.7 & 4.3 & Clark et al., 2005 \\
\hline Caucasians & 5.68 & 0.8 & Sato et al., 2005 \\
\hline Norwegian & 2.3 & 1.7 & Toft et al., 2006 \\
\hline Mixed (no Jewish) & 12 & 3.2 & Eblan et al., 2006 \\
\hline Chinese & 2.4 & 0 & Tan et al., 2007 \\
\hline Chinese & 4.3 & 1.1 & Ziegler et al., 2007 \\
\hline Taiwanese & 3.1 & 1.2 & Wu et al., 2007 \\
\hline Mixed (64\% Jewish) & 13.7 & 4.5 & Clark et al., 2007 \\
\hline Italian & 2.8 & 0.2 & de Marco et al., 2008 \\
\hline Brazilian & 3 & 0 & Spitz et al., 2008 \\
\hline Mixed & 2.9 & 0.4 & Mata et al., 2008 \\
\hline Ashkenazi Jews & 17.9 & 4.2 & Gan-Or et al., 2008 \\
\hline Portuguese & 6.1 & 0.7 & Bras et al., 2009 \\
\hline Greek & 6.4 (early onset11.5) & 3.0 & Kalinderi et al., 2009 \\
\hline Mixed (<10\% Jewish) & 12.6 & 5.3 & Nichols et al., 2009 \\
\hline British & 4.18 & 1.17 & Neumann et al., 2009 \\
\hline Japanese & 9.4 & 0.37 & Mitsui et al., 2009 \\
\hline Korean & 3.2 & 0 & Choi et al., 2012 \\
\hline Greek & 10.2 & 3.4 & Moraitou et al., 2011 \\
\hline Brazilian & 3.4 & 0 & Guimaraes et al., 2012 \\
\hline French & 6.7 & 1.0 & Lesage et al., 2011 \\
\hline Spanish & 9.8 & - & Seto-Salvia et al., 2011 \\
\hline \multirow{2}{*}{ Ashkenazi Jewish Non-Jewish } & 15.0 & 3.0 & \multirow{2}{*}{ Sidransky et al., 2009} \\
\hline & 3.0 & 1.0 & \\
\hline Polish(early onset) & 4.3 & - & This report \\
\hline
\end{tabular}




\section{References}

1 Nalls MA, Duran R, Lopez G, Kurzawa-Akanbi M, Kurzawa-Akanbi M, et al. (2013) A multicenter study of glucocerebrosidase mutations in dementia with Lewy bodies. JAMA Neurol 70: 727-735.

2 Goker-Alpan O, Lopez G, Vithayathil J, Davis J, Hallett M (2008) The spectrum of parkinsonian manifestations associated with glucocerebrosidase mutations. Arch Neurol 65: 1353-1357.

3 JamrozikZ, Lugowska A, Slawek J, Kwiecinski H (2010) Glucocerebrosidase mutations p.L444P and p.N370S are not associated with multisystem atrophy, progressive supranuclear palsy and corticobasal degeneration in Polish patients. J Neurol 257: 459-460.

4 Segarane B, Li A, Paudel R, Scholz S, Neumann J, et al. (2009) Glucocerebrosidase mutations in 108 neuropathologically confirmed cases of multiple system atrophy. Neurology 72: 1185-1186.

5 Mitsui J, Matsukawa T, Sasaki H, Yabe I, Matsushima M, et.al. (2015) Variants associated with Gaucher disease in multiple system atrophy. Ann Clin Transl Neurol 4: 417-426.

6 Zhang X, Bao QQ, Zhuang XS, Gan SR, Zhao D, et al. (2012) Association of Common Variants in the Glucocerebrosidase Gene with High Susceptibility to Parkinson's Disease among Chinese. Chin J Physiol 55: 398-404.

7 Moraitou M, Hadjigeorgiou G, Monopolis I, Dardiotis E, Bozi M, et al. (2011) $\beta$-Glucocerebrosidase gene mutations in two cohorts of Greek patients with sporadic Parkinson's disease. Mol Genet Metab 104: 149-152.

8 Lesage S, Anheim M, Condroyer C, Pollak P, Durif F, et al. (2011) French Parkinson's Disease Genetics Study Group. Large-scale screening of the Gaucher's disease-related glucocerebrosidase gene in Europeans with Parkinson's disease. Hum Mol Genet 20: 202-210.

9 Wang Y, Liu L, Xiong J, ZhangX,ZhangX, etal. (2012) Glucocerebrosidase L444P mutation confers genetic risk for Parkinson's disease in central China. Behav Brain Funct 10: 57.

10 Bodamer OA, Hung C (2010) Laboratory and genetic evaluation of Gaucher disease. Wien Med Wochenschr 160: 600-604.

11 Nichols WC, Pankratz N, Marek DK, Pauciulo MW, Elsaesser VE, et al. (2009) Mutations in GBA are associated with familial Parkinson disease susceptibility and age at onset. Neurology 72: 310-316.

12 Latourelle JC, Pankratz N, Dumitriu A, Wilk JB, Goldwurm S, et al.
(2009) Genomewide association study for onset age in Parkinson disease. BMC Med Genet 10: 98.

13 Chahine LM, Qiang J, Ashbridge E, Wilk JB, Goldwurm S, et al. (2013) Clinical and Biochemical Differences in Patients Having Parkinson Disease With vs Without GBA Mutations. JAMA Neurol 70: 852-858.

14 Winder-Rhodes SE, Evans JR, Ban M, Mason SL, Williams-Gray CH, et al. (2013) Glucocerebrosidase mutations influence the natural history of Parkinson's disease in a community-based incident cohort. Brain 136: 392-399.

15 Uchiyama A, Tomatsu S, Kondo N, Suzuki Y, Shimozawa N, et al. (1994) New Gaucher disease mutations in exon 10: a novel L444R mutation produces a new Ncil site the same as L444P. Hum Mol Genet 3: 1183-1184.

16 Beutler E, Gelbart T, West C (1990) The facile detection of the nt 1226 mutation of glucocerebrosidase by , mismatched' PCR. Clin Chim Acta 194: 161-166.

17 Hu FY, Xi J, Guo J, Yu LH, Liu L, et al. (2010) Association of the glucocerebrosidase N370S allele with Parkinson's disease in two separate Chinese Han populations of mainland China. Eur J Neurol 17: 1476-1478.

18 Clark LN, Nicolai A, Afridi S, Harris J, Mejia-Santana H, et al. (2005) Pilot association study of the beta-glucocerebrosidase N370S allele and Parkinson's disease in subjects of Jewish ethnicity. Mov Disord 20: 100-103.

19 González-Del Rincón Mde L, Monroy Jaramillo N, Suárez Martínez Al, Yescas Gómez P, Boll Woehrlen MC, et al. (2013) The L444P GBA mutation is associated with early-onset Parkinson's disease in Mexican Mestizos. Clin Genet 84: 386-387.

20 Winner B, Jappelli R, Maji SK, Desplats PA, Boyer L, et al. (2011) In vivo demonstration that alpha-synuclein oligomers are toxic. Proc Natl Acad Sci U S A 108: 4194-4199.

21 Yang NY, Lee YN, Lee HJ, Kim YS, Lee SJ (2013) Glucocerebrosidase, a new player changing the old rules in Lewy body diseases. Biol Chem 394: 807-818.

22 Dunning CJ, George S, Brundin P (2013) What's to like about the prion-like hypothesis for the spreading of aggregated $\alpha$-synuclein in Parkinson disease? Prion 7: 92-97.

23 Yap TL, Velayati A, Sidransky E, Lee JC (2013) Membrane-bound $\alpha$-synuclein interacts with glucocerebrosidase and inhibits enzyme activity. Mol Genet Metab 108: 56-64. 